\title{
Deciding how to treat anterior sectors with missing teeth
}

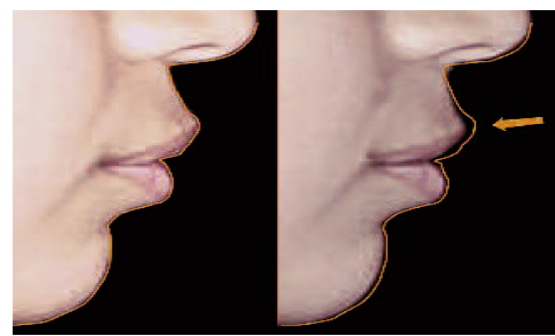

\section{Michel LE GALL, Christopher BACHET, Cyril DAMERON}

\author{
ABSTRACT \\ orthodontist.

\section{KEY WORDS} \\ Agenesis, \\ Orthodontics, \\ Prosthesis, \\ Opening, \\ Closing.
}

This article presents solutions to problems posed by missing teeth in the anterior sector of the arch by synthesizing three groups of parameters, those related to patients, those related to dentists, and those related to the

But the patient, or the parents of patients who are young children, in consultation with the treatment professionals, must make the final decision.

\section{1 - INTRODUCTION}

Teeth missing from the anterior section of the arch, either because of agenesis or trauma, pose special problems for treating dentists, orthodontists, and patients, as well, both functionally and esthetically.

The patient's smile and anterior guidance are each affected and each must be restored.
The treating team and the patients have two choices for dealing with the problem. Should they make or maintain sufficient space in the site of the missing tooth or teeth for prosthetic replacements by orthodontically creating a secondary space more posteriorly or should they use orthodontic force to close the anterior space completely?

Conflict of interests declared by the author: NONE Article received: 10-2012 M. LE GALL,

Responsable hospitalo-universitaire de l'UF d'ODF, Hôpital de la Timone, Marseille.

Villa Gabrielle,

314, boulevard Marcel-Pagnol,

13400 Aubagne.

msjlegall@aol.com

Article available at http://Www.jdao-journal.org or http://dx.dol.org/10.1051/0dten/2012204
Accepted for publication: 01-2012 
Patients are free to make this decision themselves provided the dentists involved are able to execute it correctly. If this is not possible, the patient, or parents, will have to accept an alternative solution that is technically feasible.

\section{2 - OPENING OR CLOSING: ADVANTAGES AND DISADVANTAGES}

The advantages and disadvantages of the two options of opening space for a replacement or closing it entirely can be compared in relation to these four important points:

- Esthetically the opening of space would appear to have a clear advantage because it restores the original number of teeth with each tooth taking its accustomed place in a balanced smile; the canine, a pillar tooth in the arch, can retain its normal color, volume, and shape and the coloration of the six anterior teeth can be ideal (Fig. 1). But to achieve these benefits the treatment team will have to utilize prosthesis and with it lose the supposed esthetic advantage (Fig. 2).

Conversely, while the option of closing would appear generally less advantageous esthetically (Fig. 3) it

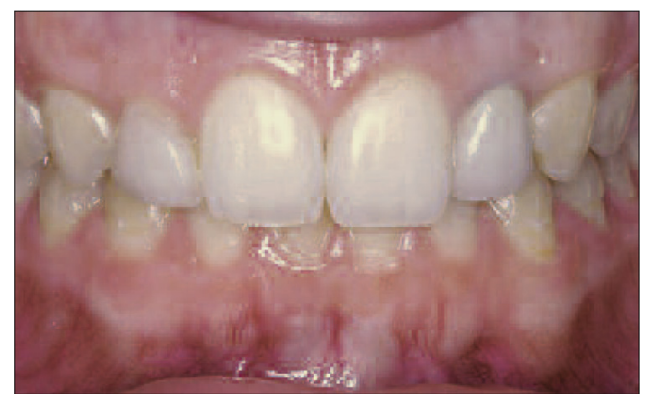

Figure 1

Filling the space left by the congenital absence of an anterior tooth with a prosthetic replacement can turn out to be quite satisfactory esthetically. can turn out to be clinically quite acceptable (Fig. 4).

In our experience and from reports in the literature, the majority of patients for whom upper anterior spaces were closed are satisfied with the result ${ }^{3}$.

\section{- From a functional point of view} opening space would seem to be preferable.

If the patient's occlusion is Class I, by opening the space the orthodontist would move the canine distally into normal position, thus respecting its role as the keystone of the arch placed in a good working position; the natural incisors and the prosthetic replacement will provide anterior guidance.

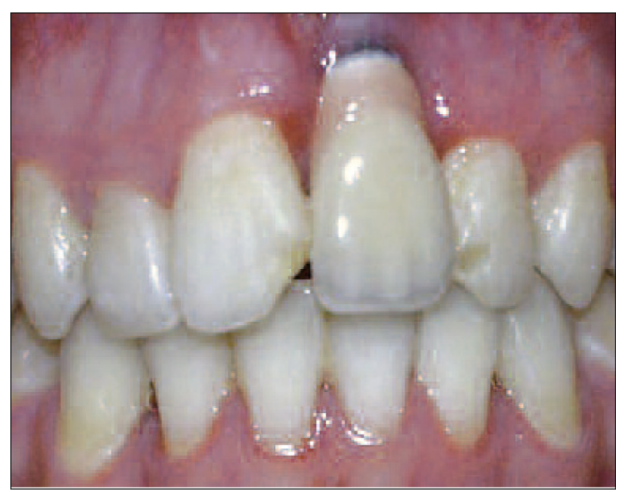

Figure 2

The upper left central incisor lost during an accident has been replaced with an implant but the loss of crestal height and the implant's emergence profile made it impossible for the treatment team to achieve a pleasing esthetic result. 


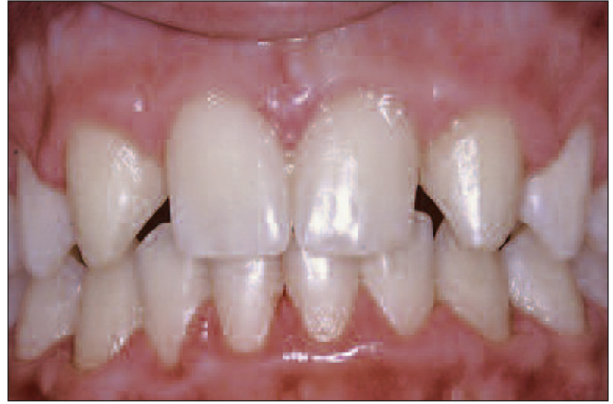

Figure 3

After the space left by agenesis of the upper laterals was closed orthodontically, the triangular shape of the canines that replace them disrupts the harmony of the anterior bloc of teeth.

But after orthodontic space closure, interferences with functional occlusion are set up. When the original occlusion was Class I, space closure requires moving the posterior segment into a Class II position (Fig. 5), unless, of course, it has been feasible to perform a compensatory lower extraction.

When an upper left cuspid has been moved forward into the lateral position, from a dynamic point of view laterality will be assured by group function in which the canine and the first premolar participate (Fig. 6).

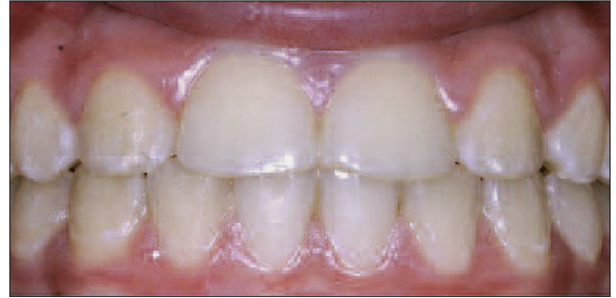

Figure 4

Here the harmony of form is respected in a similar situation by the crowns placed on the canines masquerading as laterals.

In the framework of this therapeutic Class II relationship, the orthodontist must anticipate the possibility of mesial molar rotations, and slight bicuspid rotations as well the need for grinding away some of palatal surface of the canines and some of the palatal cusps of the first bicuspids (Fig. 7).

- The prognosis for closure of spaces left by missing teeth is good if the diagnosis that indicates this option had been scrupulously carried out with regard to the dental parameters and the patient's wishes, as we shall see. But like any treatment, the prognosis is guarded if the decision maker has forced a treatment plan based more

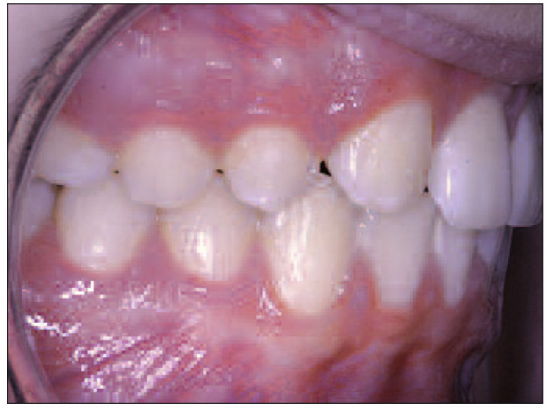

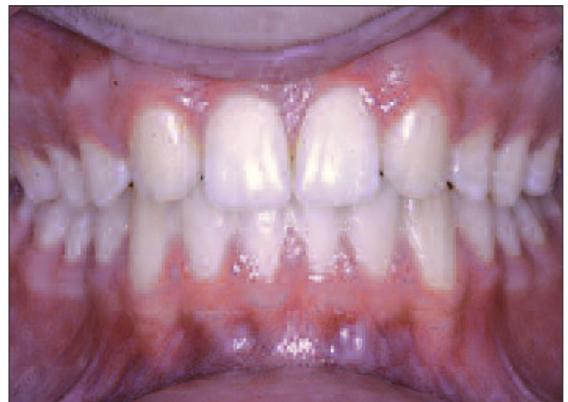

b

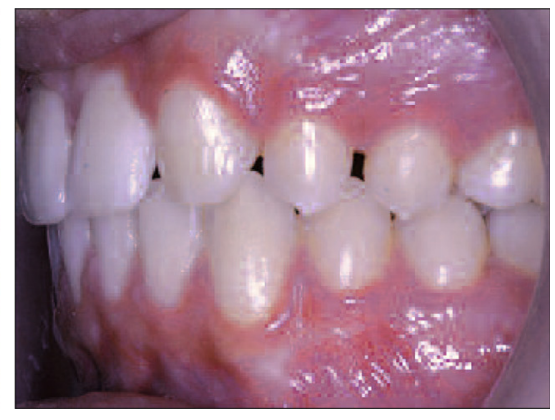

Figures $5 a$ to $5 c$

Closing the spaces left by the congenitally absent upper lateral incisors requires leaving molars in a therapeutic Class II relationship unless it is feasible to perform compensatory lower extractions. 

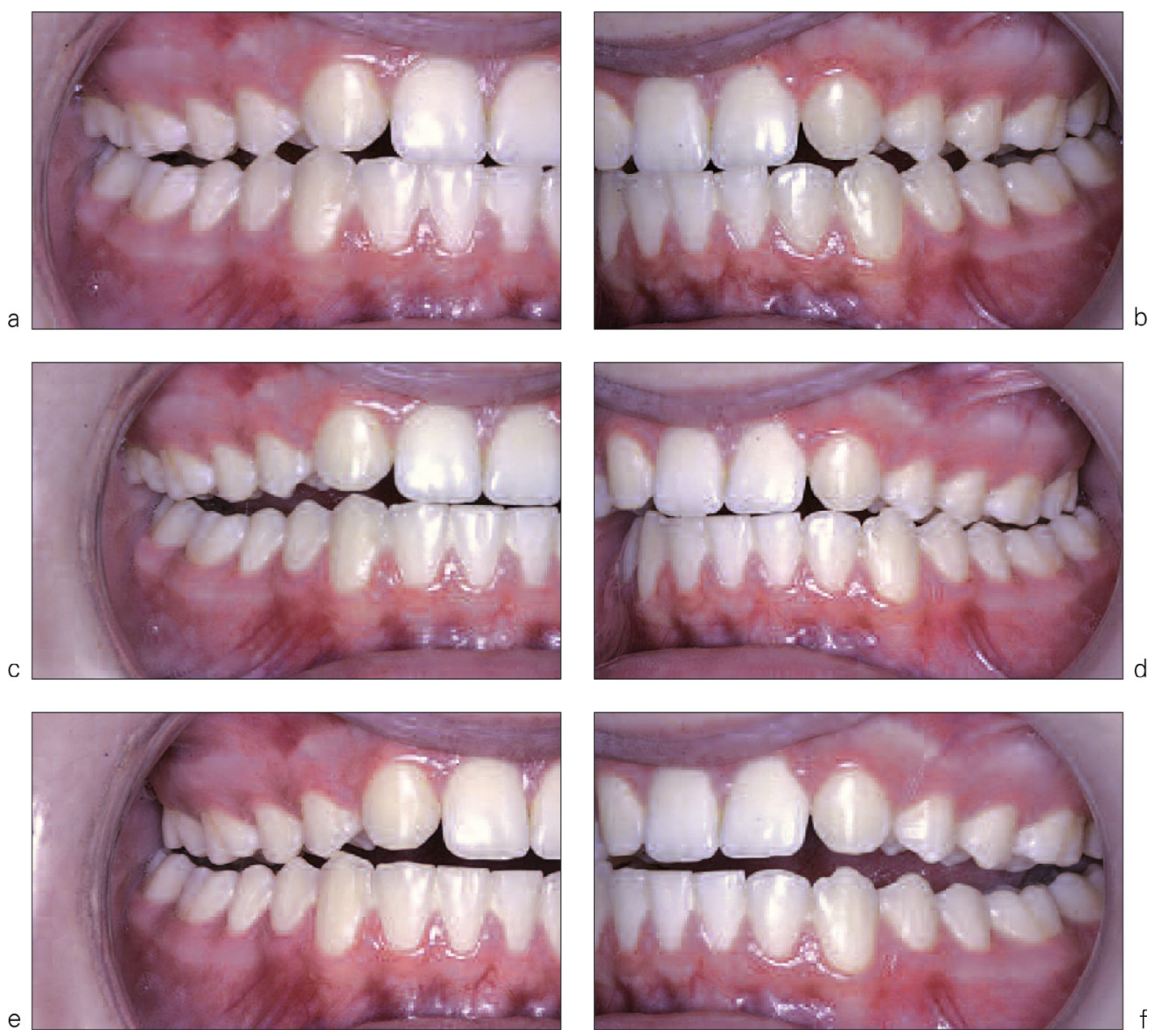

Figures 6a to $6 f$

In propulsive movements posterior teeth are out of occlusion (a and b).

Group function in left lateral excursion can be seen (c and d).

In right lateral excursion the right first bicuspids support group function (e and f).

on personal desire, financial considerations, or fear of surgery rather than on firm data. It is somewhat difficult to assess the prognosis for the opening or guarding of spaces left by missing teeth, depending as it does on all the parameters that affect the closing choice but also on other criteria. But we can state that if the patient is well and fully informed and understands all the prosthetic and surgical aspects and if a reliable and esthetic retention is assured during the pre-prosthetic period, the prognosis will be good.

- Financial criteria have been already discussed but we should add, if cost is the highest priority closure is the most advantageous option but even with this route patients should be fully aware that additional fees may be incurred if upper canines serving as laterals cannot be sufficiently reshaped to satisfy esthetic requirements. They then will require facettes. 


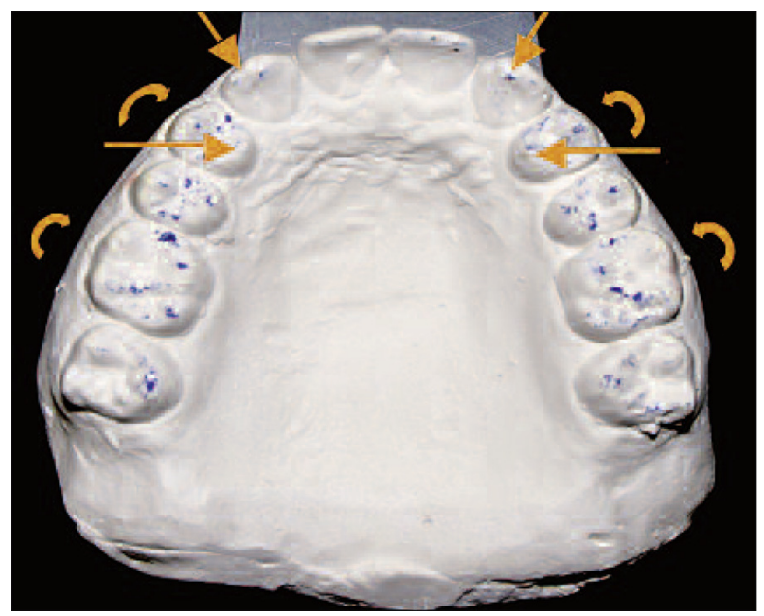

Figure 7

The points of occlusal contact objectify premolar and canine areas where crown material will have to be removed.

\section{3 - DECISIONAL FACTORS RELATED TO PATIENTS}

In deciding whether to open or close spaces left by missing upper anterior teeth, seven characteristics of patients should be considered: their age, the esthetics of their visage, the evidence provided by their X-rays, the esthetics of their dentitions, the existing occlusal relationships, their periodontal status, and any special considerations.

- Age is a preponderant factor in our choice of therapy. We distinguish three stages, child, adolescent, and adult.

By adult we mean a person whose growth has terminated and whose stage of development of osseous and periodontal tissues is at total maturity. For these patients prostheses can be constructed immediately after termination of orthodontic treatment and for whom the option of closure is rarely the treatment of choice.

For adolescents we must determine when their growth period has been or will be completed in order to predict how long the retention period will have to be because at this stage the risk of crestal bone loss is great when retention is prolonged.

Many studies have been conducted regarding this estimation ${ }^{4}$ : Behrents's study focused on chronological age, but turned out not to be reliable; the study of Vidra and Pancherz used radiographs of the hand or wrist to reveal the status of the diaphysis/ epiphysis suture of the radius to estimate residual growth within a three year plus or minus range; Nanda's study concluded that facial growth was equivalent to statural growth; Spears's study was based 
on superimposed tracings of profile cephalograms taken at 6 month intervals to measure changes in anterior facial height.

If the patient has terminated the growth period, the option of space opening can be considered favorably partly because the phase between the end of orthodontic treatment and the beginning of prosthetic therapy will be short thus reducing the risk of bone loss and unwanted tooth movements that could compromise the future prosthesis.

One of the difficulties in resolving the problem of anterior tooth loss in children is to make decisions, like, for example, extracting certain deciduous teeth, that will prematurely force a decision before some important parameters, such as coloration and form of the permanent canine, can be discerned.

Thus the necessarily long waiting period contributes to the difficulty in making the opening of space the treatment modality, because, as Beyer $^{1}$ has pointed out an option of opening cannot be made before patients have reached the age of 13 in order to avoid the risk of osseous atrophy.

As for the closing option, it is difficult to make a decision in its favor before eruption of the upper canines.

So, for younger patients, watchful waiting is often advisable until enough clinical decisional elements are in place to make an informed choice possible.

- Assessment of the esthetics of the visage is largely based on a careful study of frontal and profile photographs. Important points to evaluate include the relationship between the nose, the lips, and the chin; the volume of the lips; the antero-posterior skeletal type; the type of growth rotation; and heights of various facial sectors.

If the nose/lips/chin relationship shows that the profile of the lower third of the face is concave, space closure is contra-indicated (Fig. 8).

Lip volume is an important factor to assess because space closure provokes a retreat of the lips that is exaggerated if lip contours are delicate. But, on the other hand, overly prominent lips are a strong indication for space closure (Fig. 9).

Input from antero-posterior relationships includes assessment of mandibular retrognathy that requires a postponement of decision making until after the malocclusion is corrected. If the Class $I I$ is primarily caused by a maxillary protrusion,

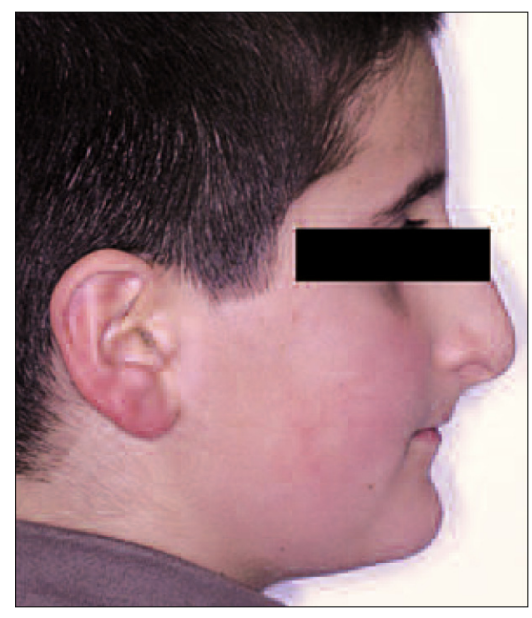

Figure 8

The nose and chin are prominent in this profile and space closure of upper anterior spaces would have a tendency to allow the lips to drop back even further thus aggravation the appearance of dental retrusion. Space opening is, accordingly, indicated. 


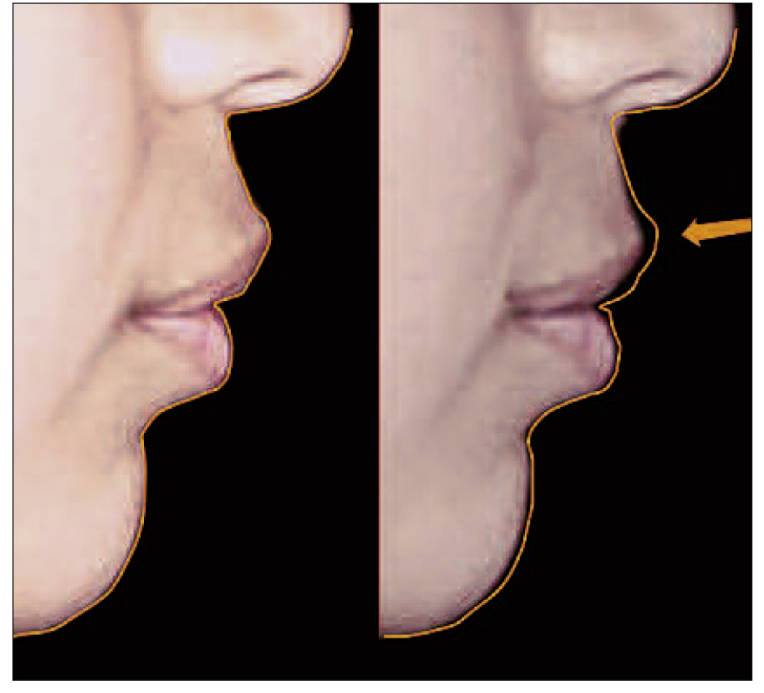

Figure 9

Space closing has stimulated a retreat of the upper lip thus improving the upper lip's overly prominent appearance.

closing is indicated because it reduces that maxillary protrusion and improves the appearance of the lips.

In Class III cases, the cause may be primarily retrusion of the premaxilla in which case opening is preferable as it is in instances of mandibular protrusion. This solution promotes the recovery of anterior guidance in a compensatory schema, surgery being excluded as a complementary procedure.

Schemas of anterior rotational growth contra-indicate space closure as much for esthetic reasons as for technical considerations.

In cases of posterior rotations, practitioners may choose either closing or opening options except in instances where opening would require distalization of buccal teeth because this could aggravate the rotation schema.

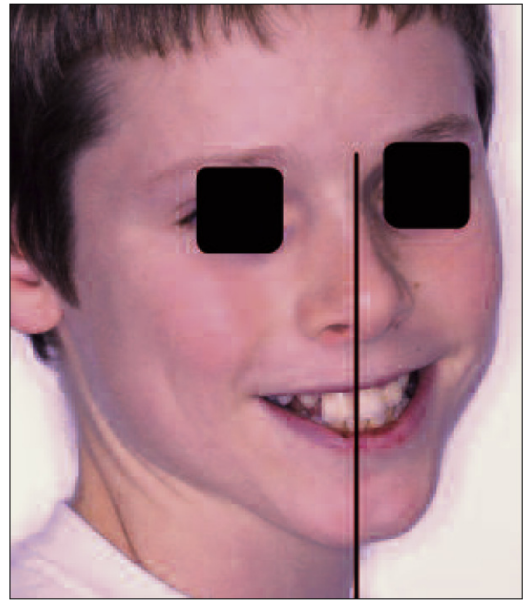

Figure 10

This patient's upper right lateral was congenitally absent. The space had closed and the maxillary midline had drifted to the right with respect to the sagittal midline.

By studying facial photographs, practitioners can observe the relationship between the sagittal midline and the midline of the upper teeth.

When one or more maxillary teeth are absent, these midlines will often not agree owing to drifting of the remaining anterior teeth into the available space (Fig. 10).

This makes the re-centering of these two midlines a primary esthetic imperative that can be accomplished by re-opening the space, that is by moving the migrated teeth back to their original positions if the diagnosis had suggested a space opening option as the preferable course or by recentering the maxillary incisors in a space created in the opposing sector. This would mean selecting the solution of extracting the erupted incisor whose mate was congenitally absent. 
- Radiography is a complementary examination that is extremely helpful to practitioners in making therapeutic decisions in cases of agenesis of teeth.

The profile cephalogram corroborated our clinical impression in this case of an overjet and overbite and a rotational schema and the panoramic film confirmed our judgment of the agenesis of the maxillary right lateral incisor and also showed us the axial inclination of the erupting upper canines as well as the degree of resorption of the remaining temporary teeth.

We were, accordingly, able to predict or orient our therapeutic choice of opening or closing by beginning serial extraction of certain temporary teeth (Fig. 11).

- Dental esthetics is no longer the determining factor in making the
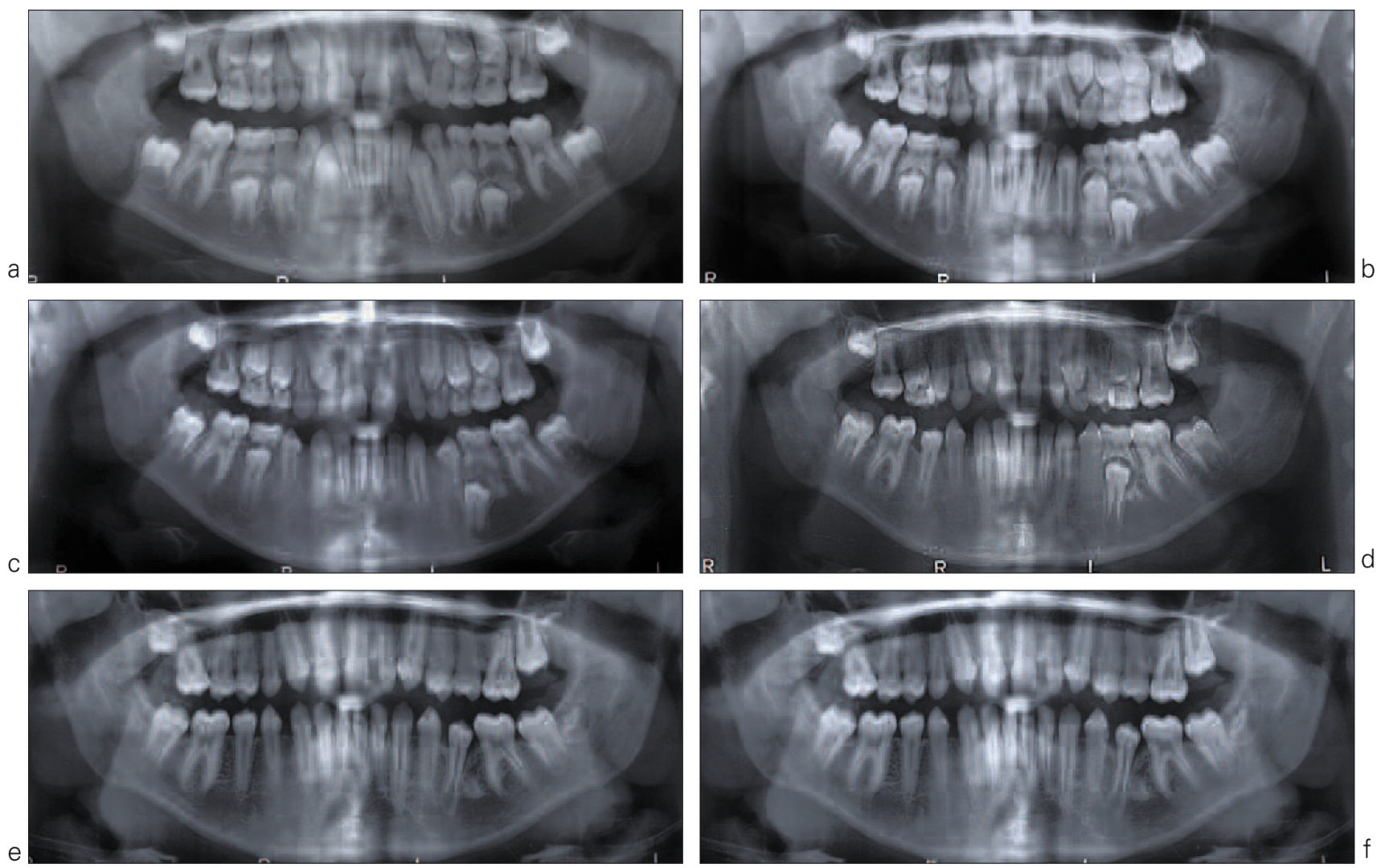

Figures 11a to $11 f$

Most of the diagnostic elements in this case of agenesis of the upper right lateral incisor suggested that opening space for its replacement should be the treatment of choice, but the patient's young age persuaded us that it would be preferable to allow the upper right cuspid to erupt in the place of the missing tooth while the temporary maxillary right cuspid served as a space retainer. When the patient is old enough for placement of an implant, we can extract the temporary cuspid and retract the permanent cuspid into its proper position after it had served to preserve the alveolar crest in the lateral site making conditions ideal for placement of an implant As a plus, the patient will have been spared the difficulties of retaining space in an area prematurely left open. 


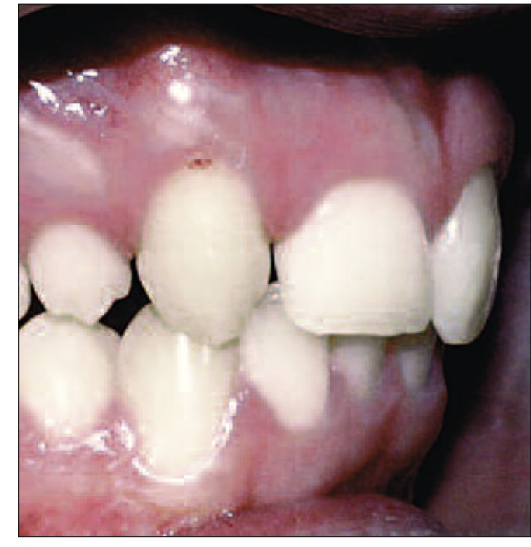

a

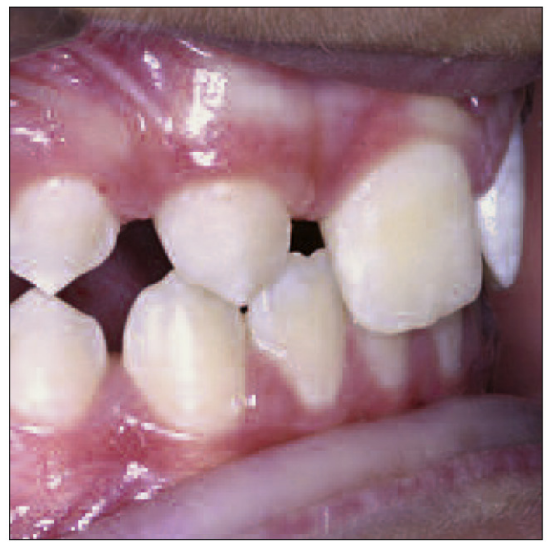

b

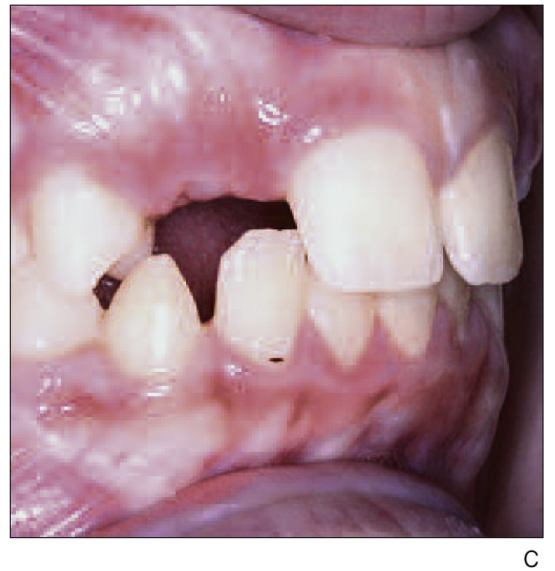

C

Figures $12 a$ to $12 \mathrm{c}$

The difference in coloration between central and cuspid is great enough to make separating them by space opening the treatment of choice. However, well made facettes could eliminate this problem and make closure acceptable.

treatment choice because modern prosthetic techniques have made it perfectly possible for practitioners to lend a pointed, bulging canine the shape and coloring of a lateral incisor.

Without reviewing all the possibilities introduced by placing of facettes, we can say that three major possibilities are available to us. The difference in coloration between canine and central may be so great that their being positioned next to each other would be unpleasing or their coloration would be similar enough that such a close relationship would be acceptable.

Finally, in some cases the upper canine teeth might not yet have erupted into the arch so the necessary reservations will be thought out and future facettes planned if the decision is made at this stage to close spaces (Fig. 12c).

The shape of the upper canines has to be taken into account. They may be rounded, triangular, large, or and, in fact, have many different anatomical configurations (Fig. 13a). The more rounded they are, the easier they are to reshape (Fig. 13b to $13 d$ ) and, inversely, if they are triangular, their greatest width will be at peak of the papilla and grinding off the peak of the cusp will not suffice to confer a laterallike appearance to it. With these teeth, practitioners must consider opening space as an attractive alternative or else using composites or even crowns to provide the desired shape.

\section{- Occlusion as a major decisional factor}

Diastemas, occlusal relationships, occlusal interlocking, arch length discrepancies, and the transverse sense are all factors to take into consideration.

Diastemas are a useful factor favoring the opening option and the larger they are, the more favorable they are. In closing them the orthodontic can concentrate the spaces where they will be needed, as sites for prosthetic teeth. 


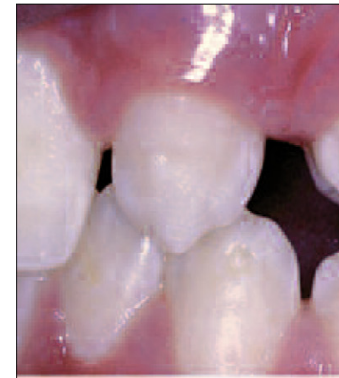

Rounded

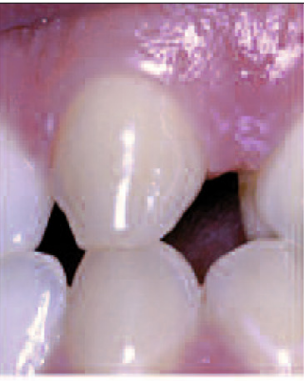

ロ
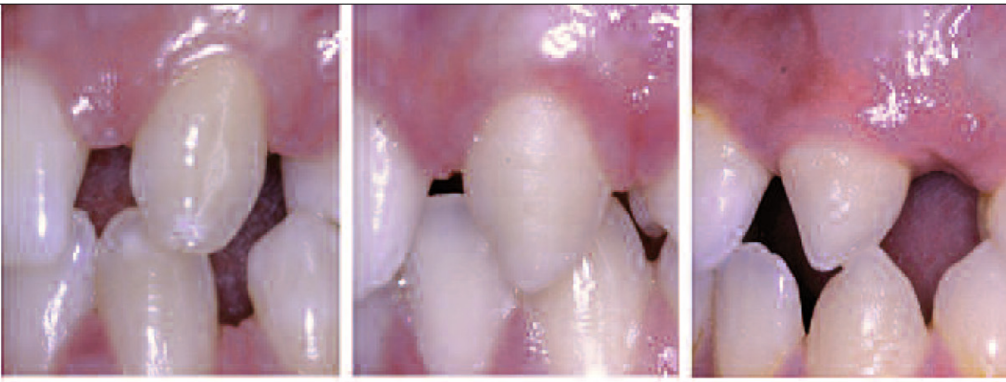

\section{Triangular}

Figure 13a

On extremely round shaped canine teeth, simply grinding off the tip of their cusps will not give them the appearance of lateral incisors. Only a prosthetic restoration can make them acceptable substitutes, so opening of space becomes the treatment of choice.
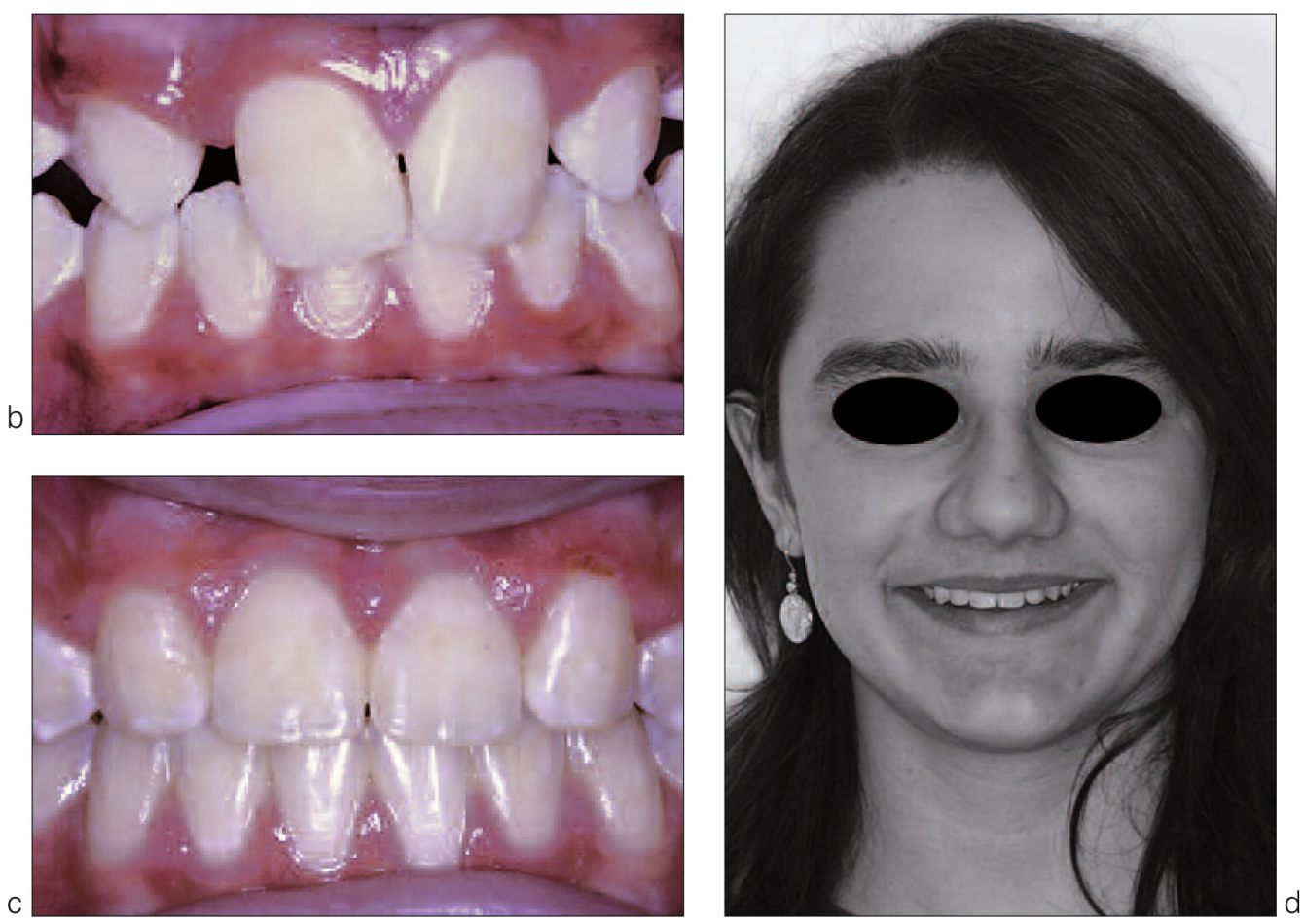

Figures $13 b$ to $13 d$

The upper canines are rounded but their coloration is similar to that of the central incisors so a simple reshaping made them acceptable substitutes for the missing lateral incisors and the closing option was indicated. 
On the other hand, if teeth have drifted to close spaces naturally, it usually makes good sense to leave them in that state of equilibrium.

In our view, occlusal relationships are probably the most important factor in deciding how to treat the spaces left by congenitally absent teeth.

If the premolars are in a Class I relationship, opening spaces is probably the best choice because closing the lateral space or spaces would mean demanding scrupulous cooperation from the patient in wearing intermaxillary elastics as an aid in mesializing all posterior teeth starting with the first bicuspids with the risk of putting the anterior teeth in crossbite.

But a Class II premolar relationship would indicate to us that closing spaces would be the treatment of choice, even though that would leave posterior teeth in a therapeutic Class II position (Fig. 14).

The extent of inter-occlusal intercuspation also influences our thinking. Preserving existing intercuspation becomes a more desirable objective if the original intercuspation is profound and, by the same token the harder it
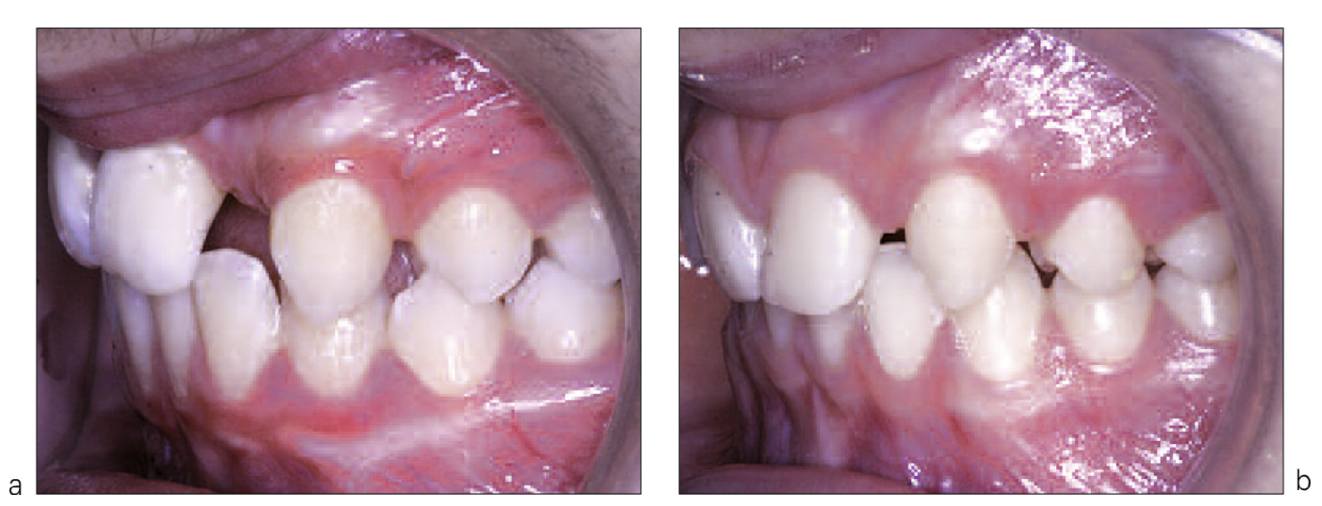

Figures $14 a$ and $14 b$

will be to overcome that intercuspation.

In modern orthodontics, however, new aids to the application of course like miniscrews, sliding mechanics, and osteoclastic blocking polarization are available to help in controlling and palliating difficult orthodontic traction.

When an arch length discrepancy accompanies agenesis of upper lateral incisors a space closing option accompanied by the compensatory extraction of selected lower teeth may be the best plan.

And if patients with congenitally absent maxillary laterals have narrow palates, rapid palatal expansion may be advisable. This increase of maxillary arch perimeter may be a factor favoring space opening.

- Periodontal considerations.

Three points are important to evaluate: status of oral hygiene, esthetics, and the prognosis for placement of implants.

As in all orthodontic treatment oral hygiene is an important element. For some patients, an apparently ideal option might not be the best. Should an orthodontist risk creating ,

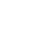

. 
precarious leucomas because of excessively long therapy?

Periodontal esthetics come into play both in the unsatisfactory relationship of the tooth collars of the canines, the central incisors, and the first premolars (Fig. 15) and the inesthetic curvature of the bodies of canines playing the role of upper lateral incisors (Fig. 16).

But the periodontal integration into the arch of prosthetic reconstructions is not necessarily ideal either even though prosthodontist and technician have been perfectly competent (Fig. 17).
We have grouped in the rubric special cases teeth requiring extraction, multiple ageneses, and cases of asymmetry.

While it is true that most anomalies of number in the upper anterior sector consist of symmetrical ageneses of the lateral incisors there are, alas, special cases of anomalies of form or resorption or a traumatic accident have obliged us to extract one or more of the maxillary anterior teeth.

In these cases, the solutions, while not ideal, were innovative and effective, utilizing, for example, a lateral incisor in place of a central incisor (Fig. 18).

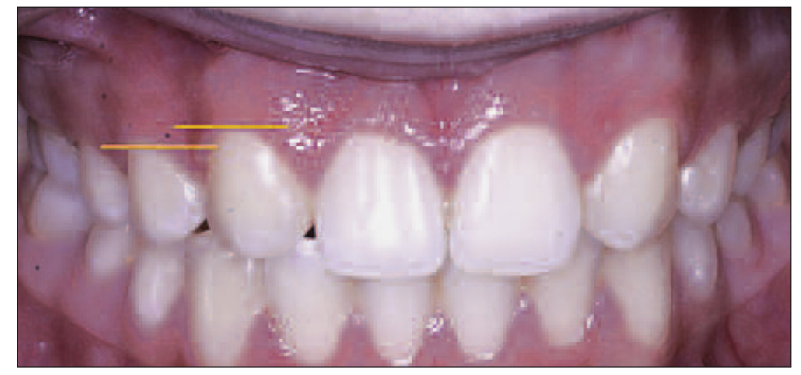

Figure 15

The first bicuspid has taken the place of the canine. Its collar should be higher than that of the lateral but, alas, the shape of the canine serving as lateral does not allow it.

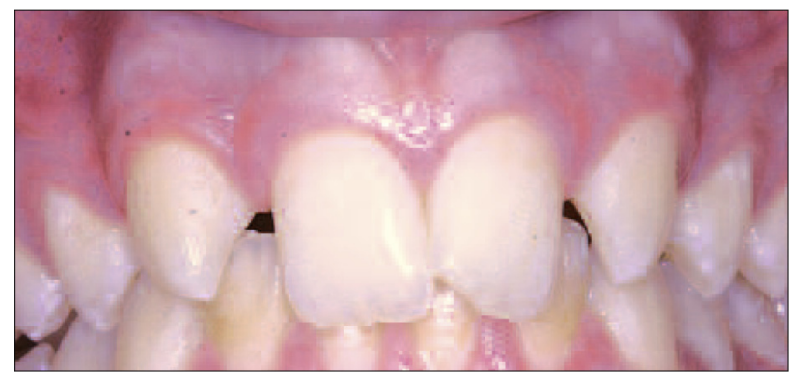

Figure 16

The rounded shape of the cuspids makes their role as laterals esthetically unsuccessful. 

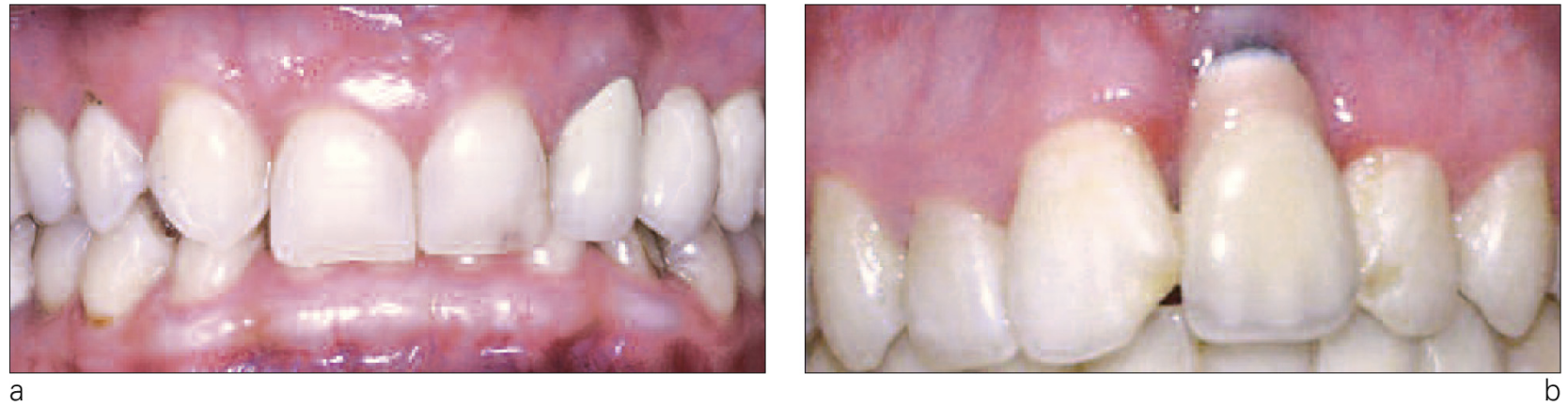

Figures $17 a$ and $17 b$

In this case of bilateral agenesis of upper laterals only the left cuspid was disguised as a lateral. The result is not esthetically preferable to the case shown in 13 a.

Crestal resorption made it impossible to integrate this implant into the arch in an esthetically satisfactory fashion (b).

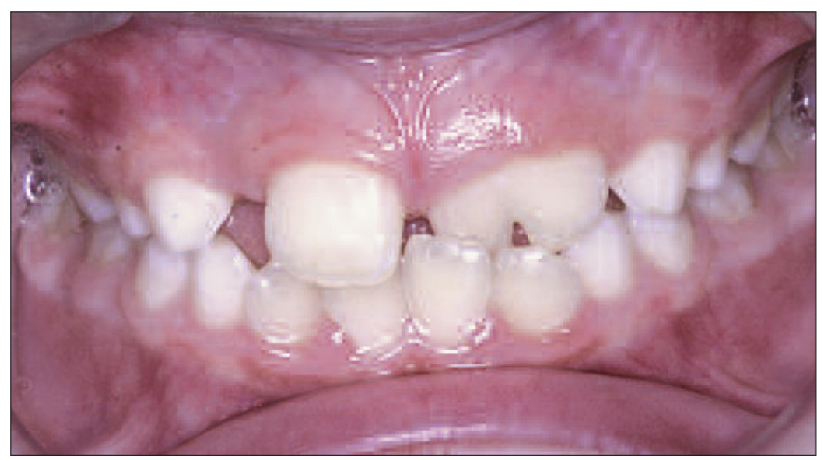

a

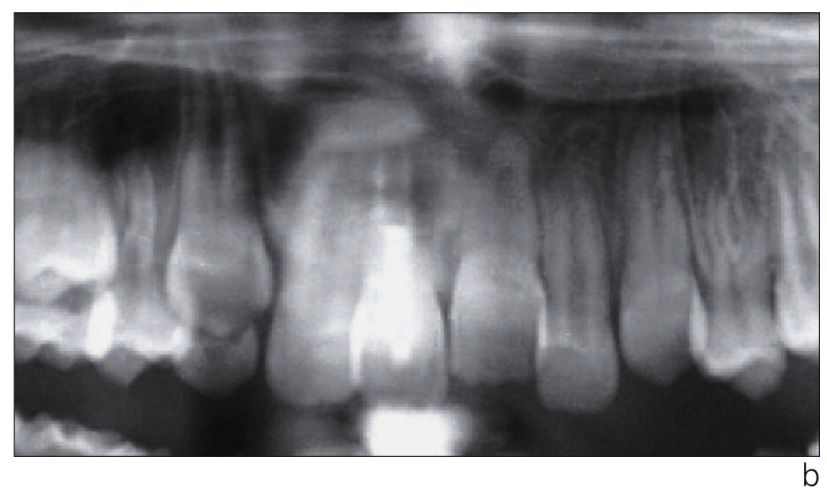

Figures $18 a$ and $18 b$

Because of taurodontism the upper left central incisor is condemned to extraction (a). The upper right central also has to be extracted because of post-traumatic root resorption (b).

Just as was the case in those situations, cases of multiple ageneses are governed by no rules; even though the broad outlines we have sketched in this article give useful suggestions on how to orient treatment, prosthodontists remain the "orchestra leaders" of these types of previously undocumented treatment (Fig. 19).

Finally, asymmetry is far from a minor problem. It requires, in fact, exquisite delicacy of management and the ability to overcome the ingrained passion of dentists to preserve all teeth at all costs because following that traditional path in cases of asymmetry can often lead to failure (Fig. 20).

As in other cases of congenitally absent teeth, the solution is either opening or closing of spaces. In this case the patient was reluctant to 

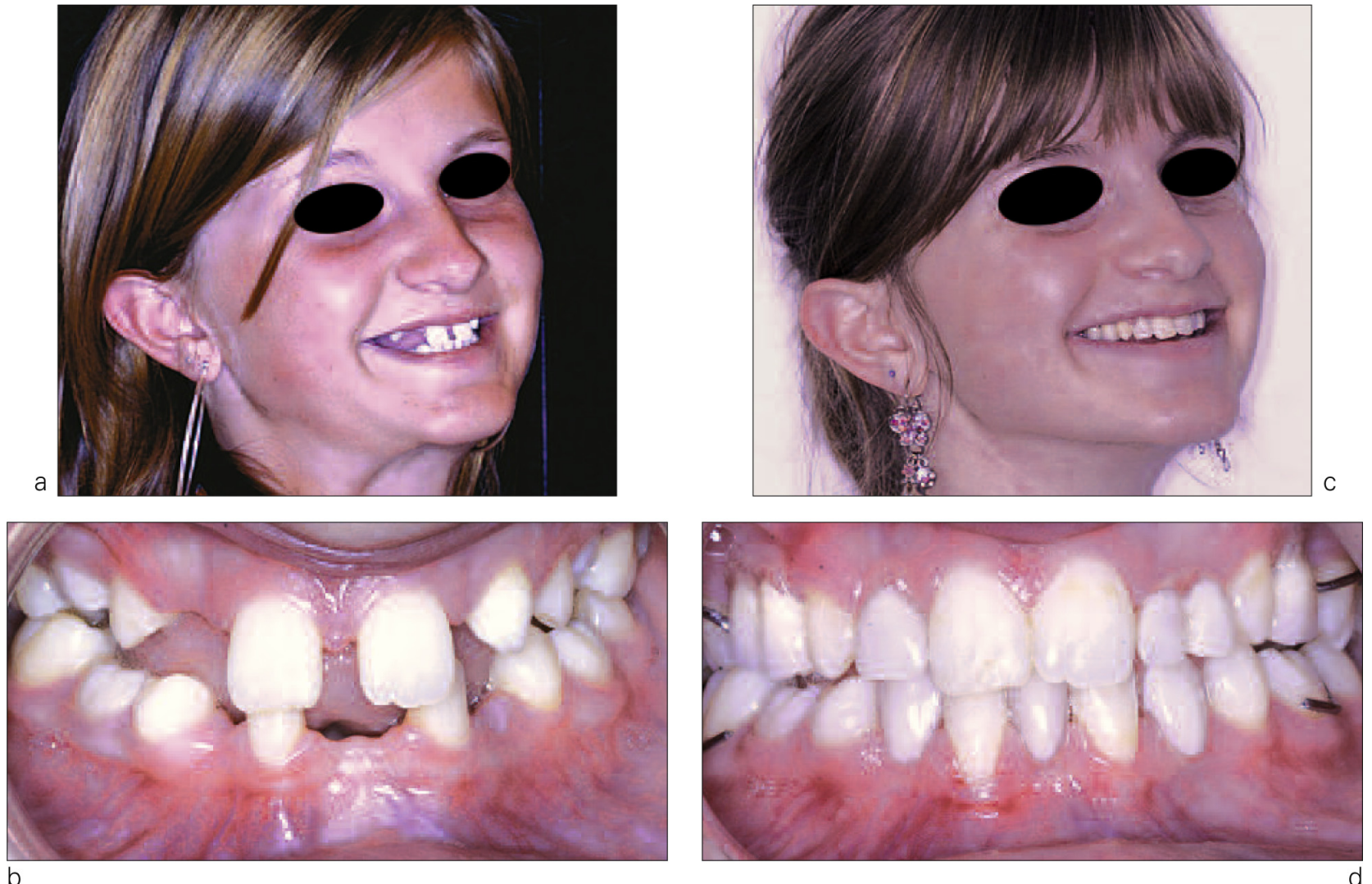

Figures 19a to $19 d$

Anterior spaces were closed orthodontically, the upper right first bicuspid was mesialized and the mandibular spaces were re-arranged in an illustration of the orthodontist at the service of the implantologist.

accept the solution of closing spaces because, for reasons of symmetry, it required the extraction of the perfectly healthy contra-lateral tooth. For other patients that decision is easier because the contralateral tooth is peg shaped. When opening is indicated it is often an ideal solution even though prosthetically reproducing a tooth perfectly symmetrical to the erupted mate is not always an easy task especially if that erupted tooth is small in size.

A meticulous assessment of all these factors led us to adopt a "made to measure" solution for this special problem. But was it technically feasible?

\section{4 - POSSIBLE TECHNIQUES AVAILABLE TO THE TREATMENT TEAM}

Can the orthodontist actually accomplish the ideal treatment plan?
If opening is indicated, can the orthodontists create the required 10 

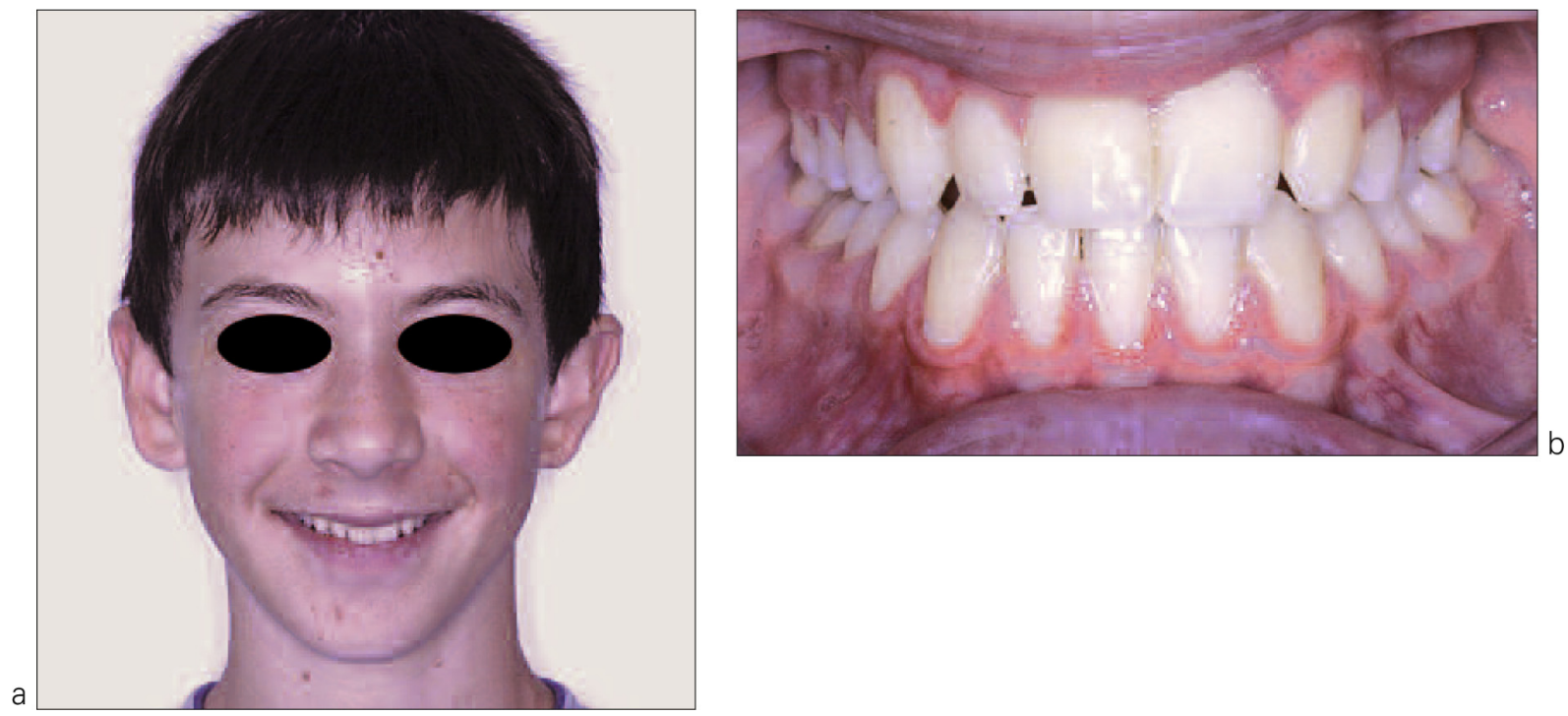

Figures 20a and 20b

Here the upper left cuspid has taken the place of the upper left lateral. The upper midline has drifted slightly toward the site of agenesis (a) Even though the upper canine posing as lateral is well integrated into the arch the difference of the height of its neck is inesthetic (b).

to 12 of space when natural tooth drifting had eliminated all the original spaces?

Or, conversely can they mesialize 8 posterior teeth, risking placement of the upper centrals into crossbite?

The answer is a guarded yes because today anchorage accessories like miniscrews, low friction full banded appliance techniques, and highly flexible memory arch wires are continuously expanding the limits of the possible. Only the biological parameters of patients, like the thickness of their alveolar bone and the quantity of their attached gingiva remain as unchanged barriers. In respecting such physiological limits treatment teams sometimes have to make concessions in their esthetic goals.

Implantologists in particular have to deal with a minefield of extremely rigorous specifications. The three-dimensional positioning of an implant, for example, demands that they conform to the mesio-distal, bucco-lingual, and vertical framework of surrounding bone, disrespect for which would inevitably lead to osseous resorption ${ }^{2}$.

In addition, as Tarnow et al. ${ }^{5}$ have shown, implantologists must leave a $1.5 \mathrm{~mm}$ osseous barrier between their artificial units and adjacent natural teeth to avoid provoking resorption.

In addition to the aforementioned requirements, we have to deal with the unique features of spaces that accompany agenesis: the absence of the tooth bud has changed the development of the supporting osseous volume, and therefore, presents a horizontal osseous deficit that is more or less noticeable, a frequent osseous 


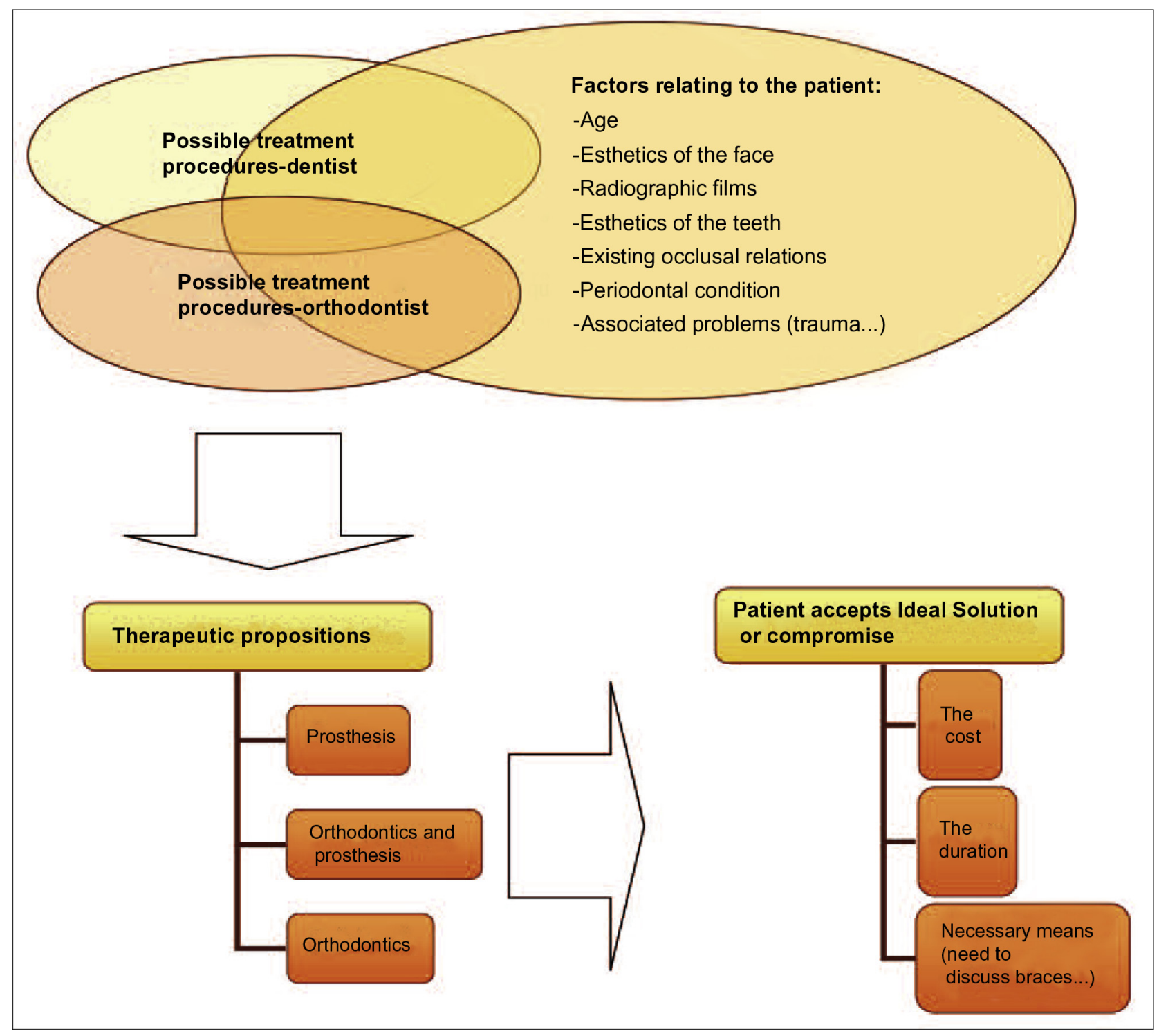

Figure 21

undercut buccally, and a flat osseous crest with a coronary excess.

The difficulties that the implantologist encounters are not systematic, but they must be planned for from the beginning because the decision to open is made well before the appropriate time for the implant.
Every option must be taken into consideration, from a bone graft to being technically blocked from performing an implant. If this is the case, what are the possible options: a bonded bridge, a conventional bridge? What definite outcome can we offer the patient? 


\section{5 - THE DECISION MAKER'S CHOICE}

The orthodontist has made a diagnosis. Taking into consideration all the "factors/patients", the decision is made whether to open or close the space created by the missing tooth.

If the choice is to open, the orthodontist speaks to the implantologist and the dental technician to plan the various stages:

- opening the space,

- the implantologist verifies that there is sufficient coronary space and implant space,

- stabilization of the orthodontic result until it is time for the implant,

- possibly repeating the pre-implant orthodontic treatment,

- possible grafts,

- performing the implant immediately if the patient is an adult,

- possibly rehabilitating the gingiva,

- installing the coronary suprastructure.

Once all the above have been done, our task is to explain and present the therapeutic option(s) to the decision maker.

If the decision maker is the patient, the "challenges" associated with implant treatment may be a turn-off.

From the perspective of the patient, all the different implant procedures might be painful and this perception

\section{CONCLUSION}

The anterior sector must have a perfect esthetic result and a perfect functional outcome.

The treatment of problems posed by missing teeth is often a compro- may deter the patient from undergoing all this discomfort. The financial factor, which is considerable for these therapeutic options, must also be added in to the patient's decision.

As a result, the main emphasis of the treatment team should be to convince the patient to opt for the ideal treatment.

However, when the patient is a child and the decision maker is the parent, there is a lack of conviction on the part of the parent because the child has to wait many years before the implant can occur.

We do not know what the base of the implant site will be many years from now. Therefore, it is necessary to plan for any eventuality, grafts etc.

The uncertainty about the future condition of the implant site makes the opening option difficult for the parent.

But when the factors/patients elements clearly indicate that opening is the better of the two options and the surgeon is optimistic about the feasibility and success of an implant, our patients will accept this option.

If there is still some hesitation, the patient will be more easily persuaded to opt for closing.

mise between numerous objective factors considered critical by the professionals and subjective factors prioritized by patients (Fig. 21). This dilemma sometimes leaves practi- 
tioners with a sense of frustration when the choice of treatment is acceptable but not necessarily ideal.

The treatment team will win the confidence of their patients by tactfully explaining possible discomfort but not letting it get out of proportion, by providing convincing reasons for what they believe is an ideal solution, and by being perfectly honest about the uncertainties involved.

\section{REFERENCES}

1. Beyer A, Tausche E, Boening K, Harzer W. Orthodontic space opening in patients with congenitally missing lateral incisors. Angle Orthod 2007;77:404-9.

2. Buatois $H$. Agénésie et traitement implantaire: l'importance de la préparation orthodontique. Rev Orthop Dento Faciale 2009; 43:43-63.

3. Robertsson S, Mohlin B. The congenitally missing upper lateral incisor. A retrospective study of orthodontic space closure versus restorative treatment. Eur J Orthod 2000;22:697-710.

4. Rozencweig G, Rozencweig S, Brun JP. Agénésie: préparation orthodontique et parodontale à l'implant. Journal de parodontologie \& Implantologie orale 2000;19: 317-31

5. Tarnow DP, Cho SC, Wallace SS. The effect of inter-implant distance on the height of interimplant bone crest. J Periodontol 2000;71:546-9. 